\title{
FRANCISCO AYALA Y EL HUMANISMO DEL EXILIO
}

\author{
INMACULADA LÓPEZ CALAHORRO
}

Universidad de Granada

\begin{abstract}
Resumen
Francisco Ayala fue editor de las revistas Realidad y La Torre, así como de la Biblioteca de Cultura Básica de la Universidad de Puerto Rico. En ellas se reseñan primeras traducciones al español de libros fundamentales sobre las antiguas Grecia y Roma, o reflexiones sobre el humanismo y su vinculación con la Antigüedad. En este artículo repasamos estas publicaciones y nos acercamos a las figuras de los autores, que compartirán el destino del exilio.
\end{abstract}

\section{Palabras clave}

Humanismo, exilio, traductores, Grecia, Roma, Francisco Ayala.

\begin{abstract}
Francisco Ayala was an editor of the magazines Realidad and La Torre and also of the Biblioteca de Cultura Basica of the University of Puerto Rico. There, he reviews first translations into Spanish of fundamental books on Ancient Greece and Rome, or reflections on Humanism and its relationship to Ancient Times. In this paper we look through these publications and pay attention to the authors who will share with him the destiny of exile.
\end{abstract}

\section{Key words}

Humanism, exile, translators, Greece, Rome, Francisco Ayala.

\section{Entre suelo y subsuelo, mientras tanto, una espesa humanidad -nosotros- se aglomera, se afana, se desvive. (Francisco Ayala, “Entre palomas y ratas", otoño de 1948).}

\section{INTRODUCCIÓN}

El exilio es un tiempo y un espacio que determina y transforma la vida de cuantos intelectuales encontraron en el continente americano la salida individual a los totalitarismos que invadieron Europa al final de la primera mitad del siglo XX. Con el exilio se sucede la paradoja de que, lejos de considerarse víctimas de tales circunstancias, parte de estos intelectuales lo interpretó como una oportunidad que los

\footnotetext{
${ }^{1}$ Correo-e: inmlop@ugr.es. Recibido: 12-09-1912. Aceptado: 11-12- 2012.
} 
liberaba de comportamientos rígidos y consensuados, de modo que se encontraban ante una oportunidad para replantearse reflexiones más libres sobre el vínculo del yo con la colectividad, o del yo con la herencia cultural e ideológica². Son las palabras del pedagogo republicano Lorenzo Luzuriaga, con quien Francisco Ayala conformará la dirección oficiosa de la revista Realidad: "Nosotros no somos exiliados. Exiliados son los que han quedado allí, sin libertad y sin posibilidades de tenerla por toda una generación" (C. Lozano Seijas apud García Montero, 2007: XXXVI).

De igual manera, Francisco Ayala, desde una posición estoica que lo protege de la nostalgia del recuerdo, vive el exilio como una oportunidad que le permite participar "íntegramente en el discurso clásico sobre el humanismo", en el que se produce la tensión entre la universalidad de principios que conforman el patrimonio humano ('homo universalis') y la conciencia de fragilidad del individuo (Navajas, 2006: 704-706). Esta clara relación con el humanismo de herencia clásica se reflejará en un diálogo directo entre los textos de la Antigüedad y el presente de estos intelectuales. La tragedia griega, el pensamiento griego o figuras romanas como Cicerón, cobrarán una actualidad que no precisará ya de la herencia vertical para justificarse. La traducción por primera vez al español de libros fundamentales para el conocimiento de la Antigüedad será un instrumento imprescindible para este cambio de relación con el pasado al tiempo que se reivindica el valor de la cultura occidental.

En este sentido, la labor que Francisco Ayala realizó como editor fue de gran valor, puesto que, sumándose al impulso de grandes editoriales como Fondo de Cultura Económica ${ }^{4}$, permitirá que se divulgue la labor de grandes intelectuales que en este período histórico aportaron reflexiones o trabajaron sobre textos de las antiguas Grecia y Roma, identificados, además, con el discurso ideológico posterior a la Segunda Guerra Mundial. Serán los nombres de Wenceslao Roces, Werner Jaeger, Karl Kerényi,

\footnotetext{
${ }^{2}$ Para esta introducción sobre el exilio y la relación con el humanismo, consideramos esencial el artículo de Gonzalo Navajas (2006), titulado "La escritura de la libertad y la cultura industrial en Francisco Ayala." Aunque nos detenemos en citas puntuales, señalamos los subtítulos del artículo: "I. Humanismo e historia cultural, II. El yo y la supraindividualidad, III. La literatura en la cultura industrial de masas, IV. Conclusión. El exilio como apertura." Asimismo, la introducción que, bajo el título "La aventura de pensar el mundo", escribe Luis García Montero (2007) para la edición facsímil de la revista Realidad.

3 "El perfil intelectual de Ayala no opera en el vacío ni le corresponde exclusivamente a él. Es propio de la figura del intelectual de entreguerras que experimenta la primera gran crisis del paradigma de la cultura moderna afiliada todavía con el humanismo clásico. [...] Para Spengler, la amenaza se concreta en la fuerza anónima y ciega de las sociedades periféricas dentro del marco occidental, es decir, aquellas culturas no conectadas directamente con el modelo greco-latino. [...] Francisco Ayala queda inserto en estos parámetros y hace una aportación específica dentro de ellos. Su posición epistemológica y ética más comprensiva se fundamenta en la independencia del yo individual frente a los modelos supraindividuales totalizantes" (Navajas, 2006: 703). Asimismo, sobre el humanismo de Ayala, cf. García Jurado (2008: 22-23) y López Calahorro (2008: 26).

${ }^{4}$ Sobre el impulso de esta editorial es fundamental la figura de José Medina Echavarría, compañero y amigo de Francisco Ayala en la Secretaría de Cortes, también exiliado con motivo de la Guerra Civil, y persona fundamental para su posterior relación con la Universidad de Puerto Rico. Echavarría en su primer destino en México se encargó de convertir a la Editorial Fondo de Cultura Económica en una editorial de prestigio (Sánchez Montes, 2006: 89), de lo que damos muestra en este trabajo por los títulos de las obras citadas.
} 
Ludwig Schajowicz, o Roger Labrousse, los que en este trabajo presentaremos como testimonio de esta cuestión.

\title{
2. BUENOS AIRES. REALIDAD (REVISTA DE IDEAS)
}

Durante su exilio en Buenos Aires (1939-1950), Francisco Ayala acepta la propuesta de dirigir una nueva revista de discusión intelectual, a la que sellamó Realidad (Revista de Ideas). Su primer número se publicó en los meses de enero-febrero de 1947 bajo la dirección de Francisco Romero, a propuesta del propio Ayala ${ }^{5}$. No obstante, en la dirección oficiosa estaban Francisco Ayala y Lorenzo Luzuriaga, miembros asimismo del consejo de redacción junto con Amado Alonso, Eduardo Mallea, Ezequiel Martínez Estrada, Julio Rey Pastor y Guillermo de la Torre. Esta revista se publicó entre enerofebrero de 1947 y septiembre-diciembre de 1949, con un total de 18 números. La idea era que la revista tuviera "sesgo ensayístico o crítico" (Ayala, 1988: 343), pero sobre todo fue el instrumento utilizado para la discusión sobre "la defensa de las raíces democráticas del pensamiento occidental” (García Montero, 2007: XLI) al tiempo que se propiciaba el hecho más cercano o coyuntural. Frente al Occidente víctima de los totalitarismos políticos, Realidad enarbola "la capacidad cultural y moral del ser humano" (ibid.). El editorial del primer número, bajo la pluma de Francisco Romero y Francisco Ayala (García Montero, 2007: XXXIX), da cuenta de la dimensión universal y de conciencia histórica que tenía la creación de esta revista de ideas:

\begin{abstract}
El Occidente debe alcanzar conciencia de sí, de sus raíces y fundamentos, de lo que en él es accidente y de lo que es esencia, de su médula viva, de sus limitaciones y de sus posibilidades. Debe también abarcar su crisis, entenderla, juzgarla, arbitrar los medios para salir de ella. Esto, en cuanto a lo que pudiera llamarse el aspecto interno. En cuanto a lo externo, debe examinar la nueva situación, abrirse a una comprensión más generosa y cabal de las otras culturas, para respetar en ellas su derecho, para incorporar aquellos de sus valores que resulten admisibles sin desmedro de la peculiaridad propia, para corregir lo que, acá y allá, hubiera de angosto y unilateral. Una cultura no se impone a quienes no la tengan por propia; únicamente es legítimo proponerla. [...] Acaso el porvenir de la humanidad en los siglos próximos -o el porvenir de la humanidad, sin más, penda en esta solemne ocasión de que la propuesta de Occidente sea aceptada. Y ello depende, a su vez, de que resulte aceptable. (Realidad, 2007: 19-20) ${ }^{6}$.
\end{abstract}

Sobre la calidad de la revista Francisco Ayala escribirá que “cuando alguna vez, por uno $u$ otro motivo, hojeo sus páginas, veo que contienen trabajos de muy

\footnotetext{
${ }^{5}$ Sobre el nacimiento de Realidad nos dice Ayala: “Quería Mallea, insistiendo mucho en ello, que el director fuera yo, pero me negué en redondo. Me negué, porque si mi repugnancia de siempre a ejercitar cualquier especie de autoridad que hubiese de imponer decisiones a mis semejantes me ha hecho rehuir a lo largo de la vida toda posición de mando, ¿cómo hubiera de haber aceptado la titularidad de ese poder irrisorio que faculta para decidir sobre la inserción de tal o cual texto en una publicación, exponiéndome -y más dada mi condición de extranjero- a las fútiles pero implacables iras de los desairados en terreno tan vidrioso como es el de las pretensiones y variedades literarias? Propuse para el cargo a Francisco Romero. [...] Luzuriaga y yo le prometimos que, en calidad de secretarios de redacción, haríamos todo el trabajo; con lo cual terminó él por resignarse a aparecer como director, prestándonos su nombre. Así fue como se hizo realidad la revista Realidad" (Ayala, 1988: 343).
}

${ }^{6}$ Sobre las referencias y citas de la revista Realidad, utilizaremos la paginación de la edición facsímil (Sevilla, Renacimiento, Colección Facsímiles de Revistas Literarias, 2007). En la bibliografía recuperamos la paginación original, así como el año de edición. 
alta calidad y que en ningún momento decayó su nivel" (Ayala, 1988: 346), o que allí confluyeron las "mejores firmas del mundo en aquellos momentos. Ingleses, alemanes, incluso portugueses" (Núñez, 2008: 175). Tales firmas concretadas en artículos o reseñas de libros sobre estudios o temáticas de las antiguas Grecia y Roma son:

J. L. R. ${ }^{7}$, “Teodoro Mommsen, El mundo de los Césares, Ed. 'Fondo de Cultura Económica', México, 1945”, Realidad, vol. I, 1, 1947, 147-148.

Lorenzo Luzuriaga, “Werner Jaeger, Paideía. Los ideales de la cultura griega, Fondo de Cultura Económica, México, 3 vol., 1942-1945”, Realidad, vol. I, 2, 1947, 307-309.

Eduardo González Lanuza, “Eco y narciso: ensayo sobre arte”, Realidad, vol. 1, 3, 1947, 325-339.

José Gaos, “El Aristóteles de Jaeger”, Realidad, vol. II, 4, 1947, 92-98.

Rodolfo Mondolfo, "Benjamín Farrington, Ciencia griega de Tales a Aristóteles, Colección Pingüino, edit. Lautaro, Buenos Aires, 1947", Realidad, vol. II, 6, 1947, 431-433.

J. L. R., "Ludwig Friedländer, La sociedad romana; historia de las costumbres en Roma, desde Augusto hasta los Antoninos, Fondo de Cultura Económica, México, 1947", Realidad, vol. III, 8, 1948, 271-272.

José M $\mathrm{M}^{\mathrm{a}}$ Chacón y Calvo, "Quevedo y la tradición senequista”, Realidad, vol. III, 9, 1948, 318-342.

Francisco Vera, “Beppo Levi: Leyendo a Euclides, Editorial Rosario, Rosario de Santa Fe, 1947", Realidad, vol. III, 9, 1948, 403-405.

Adolfo P. Carpio, “Una traducción de Plotino”, Realidad, vol. V, 13, 1949, 85-89.

Carmen Gándara, "Sócrates, la Naturalidad y la muerte”, Realidad, vol. VI, 16, 1949, 92-95.

Parte de estos intelectuales son exiliados de España o de la Europa de la Segunda Guerra Mundial. Dada la significación de algunos de los estudios o de las mismas figuras en el ámbito de los Estudios Clásicos, realizamos una breve semblanza de ellas y su aportación gracias a la existencia de Realidad, en concreto de Wenceslao Roces, Joaquín Xirau, Werner Jaeger y Karl Kerényi ${ }^{8}$.

\footnotetext{
${ }_{7}^{7}$ Iniciales de José Luis Romero. En estas referencias bibliográficas respetamos la paginación del original.

${ }^{8}$ No nos detenemos, no obstante, en la figura del insigne filósofo italiano Rodolfo Mondolfo, exiliado en Córdoba donde residió desde 1940 a 1947, y posteriormente en Tucumán, desde comienzos de 1948 hasta 1952, y donde coincidirá con otro intelectual europeo exiliado, Roger Labrousse, cuya figura trataremos posteriormente. Sólo añadir sobre la figura de Mondolfo que, considerado como un auténtico humanista y un referente de los Estudios Clásicos en Argentina, además de la reseña citada sobre la obra de Benjamín Farrington, en la revista La Torre, dirigida también por Francisco Ayala en Puerto Rico, publicará "Séneca y la infinitud del progreso espiritual" (Mondolfo, 1954: 63-74), que verá la luz el mismo año en Italia en Critica Sociale (Milano, Aprile, 1954). A Mondolfo, dada la entidad de sus estudios, Diego F. Pró le dedica un libro en dos volúmenes (1967).
} 


\section{Wenceslao Roces}

Exiliado en México y profesor de Derecho y Filosofía en la Universidad Nacional de México, Wenceslao Roces fue una de las figuras más representativas del exilio español. Destaca su labor como traductor para el Fondo de Cultura Económica y a él se le debe, entre un ingente número de traducciones, la primera versión de El capital de Karl Marx o La fenomenología del espíritu de Hegel. En cuanto a los textos relativos a las antiguas Grecia y Roma traducidos por Wenceslao Roces, en Realidad se reseñan los siguientes ${ }^{9}$ :

“Teodoro Mommsen, El mundo de los Césares, Ed. 'Fondo de Cultura Económica', México, $1945^{\prime \prime 10}$.

Es una 'nota de libros' realizada por José Luis Romero. En la reseña Romero destaca que "el título de este libro no es el de original de Mommsen, el gran historiador alemán que renovó los estudios romanos" (Romero, 2007: 165). Nos explica asimismo que, con el título El mundo de los Césares, Wenceslao Roces recoge la obra de Mommsen "Las provincias desde César hasta Diocleciano y completada su Historia Romana" (ibid.). Las provincias desde César hasta Diocleciano ${ }^{11}$, publicado en 1885, es el quinto volumen de Historia de Roma ${ }^{12}$, siendo esta traducción de Roces la primera versión en lengua castellana de este volumen. Incorpora el traductor una selección de textos de los tres primeros libros de Historia de Roma. Para Romero la selección del traductor debería haberse ajustado a la "forma original de las obras que se traducen, tanto para facilitar su consulta como para no inducir a error a los lectores" (ibid.). No obstante, el valor de la obra queda claramente expuesto, no sólo por el de la traducción y la selección, sino por poner al alcance de los lectores y estudiosos en lengua hispana una de las obras más importantes sobre la antigua Roma:

Una traducción excelente permite una lectura provechosa de este libro por tantos motivos interesantes. Y, pese a la objeción señalada, el lector culto no dejará de agradecer al editor el que haya incorporado al volumen los capítulos de la Historia Romana a que nos hemos referido. (ibid.: 166).

"Ludwig Friedländer, La sociedad romana; historia de las costumbres en Roma, desde Augusto hasta los Antoninos, Fondo de Cultura Económica, México, 1947."

\footnotetext{
${ }^{9}$ Junto a la descripción que realizamos de los libros reseñados, dada su importancia en el ámbito de los Estudios Clásicos, indicamos que de las traducciones sobre temas de Grecia y Roma, además de los expuestos, Roces realizó las siguientes traducciones: Droysen, Johan Gustav, Alejandro Magno, México, FCE, 1946 (481 p.); Gregorovius, Ludwig, Atenas en la Edad Media y otros ensayos, (Prólogo de W.R.), México, FCE, 1946 (417 p.); Mayr, Robert von, Historia del Derecho Romano, Barcelona, Labor, 1931 (2 vol.); Rohde, Edwin, Psique: la idea del alma y la inmortalidad de los griegos, México, FCE, 1948 (368 p.); Sohm, Rudolf, Instituciones de Derecho Privado Romano: historia y sistema, Madrid, RDP, 1928 (711 p.). Citamos por la página de la web de la Fundación Wenceslao Roces.

${ }^{10}$ Las referencias bibliográficas son citadas tal y como aparecieron en Realidad.

${ }^{11}$ Su título original es: Das Römische Imperium der Cäsaren. Römische Geschichte.

12 Römische Geschichte, escrita entre 1854-1856, traducida al español en 1876 por Alejo García Moreno como Historia de Roma. Para la figura de Alejo García Moreno, cf. Mirilla Romero (2005: 135-152).
} 
Su título original es Darstellungen aus der Sittengesch. Rom's in der Zeit von August bis zum Ausgang der Antonine (3 vol., 1862-71; 6th ed., 1889-90). En la revista Realidad (vol. III, 7, 1948, sin número de página) la editorial Fondo de Cultura Económica anuncia el libro de Friedländer:

Magnífica traducción de una de las obras más famosas de la literatura histórica, aparecida bajo el título de "Cuadros de historia de las costumbres romanas, hermosamente ilustrada con 42 láminas a toda página, encuadernada... \$ 40 .

Es en el número siguiente (III, 8, 1948, 403-405) donde aparece la reseña de José Luis Romero sobre el libro con la traducción de Roces. Resalta Romero que, casi un siglo después de que saliera a la luz, se traduce al español la gran obra del erudito alemán. Friedländer, seguidor de Teodoro Mommsen y de Jacob Burckhardt, escribió este libro en un momento posterior a la crisis de 1848, razón por la que los historiadores cambiaron el contenido histórico político anterior. Así nos explica Romero las características que hacen de la obra de Friedländer un texto fundamental para conocer la Antigüedad y la importancia de que por primera vez se traduzca al español, prácticamente un siglo después de su publicación:

Grandes cuadros de conjunto en los que correspondieron al pueblo un papel de primer plano, mostrando sus formas de vida y las alternativas de su actitud frente a las figuras singulares que parecían dirigir la vida social. [...]. Así surgió, de un conjunto, no muy orgánico por cierto, lo que se empezó a llamar, sin demasiada precisión, por lo demás, historia de la cultura o de la civilización.

Dentro de esta corriente, que despertaba en su tiempo, se orientó Ludwig Friedländer, y fruto de sus investigaciones en ese sentido fue su notable libro sobre la sociedad romana que acaba de publicarse en México, traducido por primera vez al español. (Romero, 2007: 1377).

\section{Wenceslao Roces, Joaquín Xirau y Werner Jaeger}

"Werner Jaeger, Paideía. Los ideales de la cultura griega, Fondo de Cultura Económica, México, 3 vol., 1942-1945."

Paideía es uno de los textos fundamentales de análisis de la cultura griega en torno al complejo concepto griego que da título al libro, cuyo alcance es mucho más amplio de lo que podríamos traducir en nuestra lengua como "educación". Publicada en Fondo de Cultura Económica, la primera edición en español de 1942 consta de un prólogo del autor alemán ${ }^{13}$. En él da gracias a Joaquín Xirau como traductor, que así lo fue de los libros I y II, pero su anticipada muerte hizo que Wenceslao Roces tradujera los libros III y IV, por lo que no es mencionado en el prólogo. La edición en tres volúmenes que reseña Romero se sucede entre los años 1942 y 1945, siendo los libros III y IV publicaciones por primera vez del original alemán también inédito y con la traducción al español. La primera edición en un único volumen será muy posterior, de 1957.

Joaquín Xirau es también un exiliado de la Guerra Civil española, de origen catalán y afincado en México. Discípulo de la Institución Libre de Enseñanza, decano de la Facultad de Filosofía de Barcelona, huyó hacia Francia en la misma ambulancia

\footnotetext{
${ }^{13}$ La primera edición en alemán, en 1933, y una segunda, en 1935, tienen también prólogos de W. Jaeger.
} 
que Antonio Machado. Representa Xirau la figura de un auténtico pedagogo que se ocupó profundamente de los métodos de enseñanza. Seguidor de su admirado Ramón Llul, escribirá citándolo: "El amor ha sido creado para pensar" (Xirau, 1996). Es, por consiguiente, un auténtico humanista, que en su exilio en México adquirió una nueva forma de mirar los estudios humanísticos, tal y como su hijo Ramón Xirau (2006) nos relata sobre su memoria:

Mi padre me dijo y dijo a varios de nosotros que en México había descubierto a España. Otra España, en efecto; la de los humanistas, la de Vives, la de Sahagún, Las Casas, Vasco de Quiroga. En todos ellos hay algo de común y de valor especialmente importante en nuestros días de desvalor, el "orden del amor".

Sobre la trascendencia de los estudios de Xirau en el ámbito de la educación, Antoni Mora reivindica su figura precisamente a la luz de la palabra griega, titulando "La paideía republicana de Joaquín Xirau" al prólogo del Tomo III de sus obras completas (Mora, 2000: XI-XX), en el que establece la relación y la diferencia entre el concepto 'educación' de Xirau y el de Jaeger. Es así que se establece una línea de comunicación indirecta entre la Antigüedad y la actitud activa filosófica, política y pedagógica del pedagogo catalán.

En cuanto al libro de Jaeger, recordamos que no es sólo un estudio sobre la antigua Grecia, sino también un análisis que trasciende la cultura europea e igualmente transmisor del compromiso humanístico de su autor. Para ello nos sirven las palabras del autor en el prólogo de la edición española, escrito en la Universidad de Harvard en julio de 1942, adonde se había exiliado el alemán huyendo del nazismo. En el prólogo, en el que hace referencia a la primera edición que correspondía al período de entreguerras, dice:

Este libro se escribió durante el período de paz que siguió a la primera Guerra Mundial. Ya no existe el "mundo" que pretendía ayudar a reconstruir. Pero la Acrópolis del espíritu griego se alza como un símbolo de fe sobre el valle de la muerte y destrucción que por segunda vez en la misma generación atraviesa la humanidad doliente. (Jaeger, 1962: X).

Desde esa mirada que sobrevuela lo que acaece en el tiempo contemporáneo del autor a causa de las dos guerras mundiales, de las que la sucesiva redacción de los libros que conforman Paideía son testimonio, es el también pedagogo y colaborador de Francisco Ayala, Lorenzo Luzuriaga, el encargado de la reseña para Realidad. De sus palabras destacamos la valoración que hace del libro de Jaeger por su actualidad:

Cuando se buscan los orígenes de las ideas e instituciones esenciales de nuestra cultura, siempre venimos a parar, por un camino $\mathrm{u}$ otro, a la fuente cristalina de Grecia. Alguien ha dicho, en este sentido, que excepto las fuerzas ciegas de la naturaleza, nada se mueve en el mundo que no sea griego en su origen. Así vemos, por ejemplo, que los ideales políticos-pedagógicos que han estado en pugna últimamente y que aún lo están en cierto modo: el totalitario y el democrático liberal tienen sus antecedentes remotos, pero manifiestos de la educación helénica: la espartana y la ateniense. A pesar de todas las aparentes diferencias, estas concepciones se corresponden de un modo tan manifiesto con aquellos ideales, que es raro que no se haya reconocido antes esa coincidencia. De aquí que el estudio de la cultura clásica tenga un valor permanente, ya que nos permite ver en su originaria pureza las ideas que predominan en la vida de nuestra época. (Luzuriaga, 2007: 347). 
Esta conciencia de la comparación entre los dos tiempos, entre el de la Antigüedad y el de la época que viven los exiliados en Argentina, producto de los conflictos bélicos que asolan Europa, es fundamental en los análisis que de la realidad hace también Francisco Ayala utilizando las referencias del mundo clásico. Cuando en 1978, casi treinta años después, escribe el prólogo para la edición de El tiempo y yo, o el mundo a la espalda, comprobamos el ejercicio de una relación semejante a la expuesta por Luzuriaga en la reseña sobre el libro de Jaeger, con la conciencia de la permanencia de la cultura clásica por su actualidad:

\begin{abstract}
Por ejemplo: lo inaudito de una noticia leída en el periódico matutino que nos ha escandalizado por lo pronto como muestra increíble de la locura de nuestros tiempos, puede llevarnos hacia un texto clásico donde lo referido en el diario encuentra su parangón. Con esto, la trivialidad del suceso publicado hoy para que mañana esté olvidado adquiere, sobre el fondo del texto latino, la dignidad de una permanencia que lo hace perenne, mientras que a su vez el texto viejo y distante, apergaminado, se nos hace actual al sufrir ante nuestros ojos la invasión de una sangre caliente. (Ayala, 2006: 16).
\end{abstract}

\title{
Karl Kerényi
}

En los números 7 y 9 del volumen III de Realidad, en 1948, se publica la conocida "Carta sobre el humanismo" de Martin Heidegger"14 (2007: 1091-1115 y 1467-1491). Entre ambas partes en que se divide el texto de Heidegger, se publicará “El humanista en la encrucijada" de Karl Kerényi (2007: 1253-1263). Sobre su figura en el artículo publicado en Realidad, en nota a pie de página se señala: "Profesor de la Universidad de Budapest, es uno de los más prestigiosos especialistas actuales en Mitología e Historia de las religiones" (ibid.: 1253). Kerényi (1897-1973), doctor en Filología clásica por la Universidad de Budapest, alumno visitante en distintas Universidades y de los prestigiosos filólogos clásicos Eduard Norden y Ulrich von Wilamowitz-Moellendorff, desertor político en 1944 cuando los nazis entraron en Hungría, víctima asimismo del estalinismo y exiliado finalmente en Suiza donde conseguirá la nacionalidad y desarrollará parte de sus estudios, es uno de los grandes eruditos de los humanistas de la Antigüedad y uno de los fundadores de los estudios modernos sobre mitología. Junto con Carl Gustav Jung escribió Introducción a la esencia de la mitología (1941).

Kerényi en "El humanista en la encrucijada”, firmado en Budapest (invierno 1947/48), destaca la contraposición entre la humanitas y lo "inhumano"15, y repasa el concepto 'humanismo' en las antiguas Grecia y Roma, para reclamar el papel esencial que ha de ejercer el filólogo clásico contemporáneo, más allá del ejercicio de la investigación histórica:

Ahora sí debe hablar el filólogo, precisamente por tener conciencia de su ocupación, no es idéntica al 'humanismo' en su sentido más amplio tal como lo definimos antes; y más aún: de

\footnotetext{
${ }^{14}$ La traducción de la obra de M. Heidegger, "Carta sobre el humanismo", se debe a A. Wagner de Reyna, publicado en Realidad ( 7-9, 1948).

15 “Hoy día, cuando hemos abandonado hasta la idea de que pueda proyectarse alguna claridad desde la humanitas hasta su polo opuesto, ha llegado la hora y la tensión en lo inhumano, en favor del humanismo como tal y para los estudios humanísticos o, mejor, lo que ha quedado en pie de estos estudios como investigación científica de la Antigüedad" (Kerényi, 2007: 1255).
} 
que esa ocupación, humanística sólo por su origen, en cuanto investigación histórica ha perdido desde hace tiempo aquel apasionado interés por el hombre que caracteriza y caracterizará al 'humanista' mucho más que el conocimiento del latín y el griego. (Kerényi, 2007: 1255).

Son reseñables sus conclusiones que relacionan el humanismo tanto por semejanza como por diferencia con su valor en la Antigüedad:

También este humanismo buscaba soluciones en la Antigüedad: soluciones de la problemática de la formación, no meramente del ser hombre. Por aquel entonces todavía no se impuso la evidencia de que en lugar del hombre puede presentarse de repente también el no-hombre. Tuvo que llegar la segunda guerra mundial para que, como alusión al menos, el gran filólogo Karl Reinhardt, formulara la presunción de que la solución clásica se origina allí donde 'se lucha por la imagen del hombre amenazada'" (Kerényi, 2007: 1263).

Uno de los elementos fundamentales de conexión con la obra de Francisco Ayala viene determinado por el uso del precepto socrático-délfico 'conócete a ti mismo'. Kerényi, a propósito de Sócrates, dice, utilizando al filólogo como sujeto:

La sentencia délfica 'conócete a ti mismo' lo remite a una cuestión que habría de resolverse antes que ninguna otra, y a la que él todavía no puede contestar: si acaso él, el hombre Sócrates, no es un monstruo más complicado y más monstruoso aún que el fenomenal Tifeo. ¿O acaso él, el hombre, es un ser más suave y más sencillo, que participa en algo divino y no-monstruoso? (Kerényi, 2007: 1258).

Esta sentencia estará presente en Francisco Ayala como principio rector (López Calahorro, 2008: 153-160). Es, quizá, el elemento fundamental con el que el intelectual refleja su compromiso vital individual, su medida humana, lo que en otro lugar hemos llamado 'humanismo inapelable' (López Calahorro, 2008: 26) ${ }^{16}$. Pero también hay otros puntos de encuentro entre el filólogo y Francisco Ayala, de modo que, al repasar Kerényi el concepto humanismo, incorpora las aportaciones procedentes de los autores griegos y romanos como Terencio, Menandro o Platón, y tras ellos aparece la gorgona Medusa:

Tenemos que leer estos tristes signos. Y con ello cobra vida el rostro de la gorgona que estaba siempre ante nuestros ojos sin que nos diéramos cuenta. Emerge, por así decirlo, y actúa. Espero que actúe de un modo bien distinto al de la mitológica cabeza de medusa: ésta paralizaba a todo lo viviente, mientras que la nueva debiera liberar lo paralizado. (Kerényi, 2007: 1260).

Publicado el texto de Kerényi en 1948, destacamos la similitud en el uso de medusa que nos expone Francisco Ayala en su ensayo "Calderón. Porque no sepas que sé", publicado en $1961^{17}$. En este texto Ayala refleja una contradicción similar a la de Kerényi sobre la acción paralizante de la gorgona, al reclamar que su acción sea liberadora en lugar de paralizadora, relatividad que en Ayala se reflejará en un doble sentido: tanto en la ambigüedad de quien es agente portador de la mirada paralizante ${ }^{18}$,

\footnotetext{
${ }^{16}$ Sobre el humanismo de Ayala: “Ahí, en esa individualidad que tan cara cuesta, es donde veo que radica el 'humanismo inapelable' de Ayala. Cicerón y Aulo Gelio hablaron sobre la dimensión de la humanitas. La medida humana se ha visto terriblemente amenazada en el siglo XX. Pero Ayala no puede ser entendido sin esa medida específicamente humana. Como ha visto Inmaculada, la mejor expresión de esta medida es el socrático (délfico) 'conócete a ti mismo' “ (García Jurado, 2008: 23).

${ }^{17} \mathrm{El}$ texto están incluido en La plumas del fénix (Ayala, 2007).

${ }^{18}$ En su ensayo "Porque no sepas que sé", refleja el aspecto contemporáneo de esta mirada de Medusa: "Segismundo quiere exterminar al intruso, quiere quebrar el espejo de la conciencia ajena, porque se siente degradado a sus ojos. La vista del extraño, del otro, es siempre mirada de Medusa, que nos petrifi-
} 
o la mirada también liberadora de la conciencia, más exactamente interrogadora, que podrá ser la cabeza del cordero, en el relato que lleva este nombre (López Calahorro, 2010).

\section{PUERTO RICO. LA TORRE Y BIBLIOTECA DE CULTURA BÁSICA}

De Argentina marcha Francisco Ayala a Puerto Rico, donde ocupará la plaza de Catedrático de Ciencias Sociales y dirigirá el Servicio de Publicaciones ${ }^{19}$. A Puerto Rico llega para dar un curso en la Universidad y allí, por encargo del rector de la Universidad, Jaime Benítez, el escritor pondrá en marcha, en colaboración con la Revista de Occidente, la colección Biblioteca de Cultura Básica de la Universidad de Puerto Rico ${ }^{20}$. También dirigirá una nueva revista, La Torre, que actualmente se sigue editando. Ambas empresas se fundarán en 1953 y en ninguna de ellas, tal y como había ocurrido en Realidad, aparecerá el nombre de nuestro escritor (García Montero, 2009: 121-122).

En La Torre destacamos la presencia de dos eruditos de la mitología moderna: Ludwig Schajowicz y Mircea Elíade. Durante el período en que Francisco Ayala dirige la revista La Torre, se publica “Orfeo y Anfión" de Schajowicz (1954) y otro artículo de mitología a cargo del Eliade, "Los mitos en el mundo moderno" (1954). Ambos títulos se presentan en números sucesivos de la revista, en concreto en los números 5 y 6, respectivamente. Sobre la figura del rumano Mircea Eliade, anotamos también su condición de exiliado con motivo de la Segunda Guerra Mundial, instalándose primeramente en París y posteriormente en Chicago. Su amplísima bibliografía da cuenta de su talla intelectual y de sus amplios e innovadores conocimientos, especialmente en el ámbito de la mitología.

En cuanto a Ludwig Schajowicz, de origen austriaco, en 1938 emigró a Cuba, donde fundó el Teatro Universitario de la Habana. Posteriormente marchó en 1947 a Puerto Rico como invitado para impartir unas conferencias, y donde se estableció hasta su muerte como Catedrático de la Universidad. Entre sus obras destacamos Mito y existencia (1962) y El mundo trágico de los griegos y de Shakespeare (1990). Mito y existencia está pensado treinta años antes de su publicación, es decir, incluso antes del

ca y convierte en objeto. ¿Quién en el mundo habrá sido tan dichoso que nunca haya sentido el impulso de aniquilar -siquiera sea a su vez con la mirada- al que nos ha sorprendido acaso en un momento de debilidad? [...] Todo esto, que en nuestros días ha puesto muy de relieve el análisis existencial, se encuentra expresado con fórmula definitiva en los dos versos de Calderón" (Ayala, 2007: 771).

19 “Jaime Benítez [...] me propuso que me quedara con un contrato permanente para organizar el curso básico de ciencias sociales. Al año siguiente me encomendó la dirección de la editorial universitaria para desarrollar un programa de publicaciones bastante amplio, dentro del cual fundé y puse en marcha una revista. La Torre, que ha sido por varios años la mejor publicación de su género en lengua castellana" (Ayala, 1988: 353).

20 “A mi llegada a Puerto Rico fui encargado de reorganizar el curso básico de ciencias sociales [...]; pero ya al año siguiente me propuso el rector que me hiciera cargo de la Editorial Universitaria y planease un programa en cooperación con la Revista de Occidente, y por cierto, que si esto comportaba un enriquecimiento cultural para aquel país, la ventaja económica fue para Ortega y su familia. Sería casi un alarde de parte mía la mera enumeración de las obras que se publicaron en un breve lapso" (Ayala, 1988: 378). 
ensayo "Orfeo y Anfión", al que citaría en su publicación posterior. En el prólogo de la edición de 1962, con fecha de abril de 1961, editado también por la Universidad de Puerto Rico, Schajowicz anota:

Este libro es la versión revisada y considerablemente ampliada de un trabajo que terminé a principios de 1958. Algunos capítulos de la primera versión aparecieron en las revistas La Torre (Puerto Rico) y Humanitas (México). (Schajowicz, 1990: 20) ${ }^{21}$.

Carlos Rojas (2005), a propósito de un estudio sobre los 'transterrados' en Puerto Rico, destaca en primer lugar precisamente al intelectual austriaco. Profesor en este país de varias generaciones de estudiantes, seguidor de Nietzsche y de Heidegger, Schajowicz restituye el pensamiento mítico griego frente al monoteísmo religioso, de modo que para él "el mito es la verdad de la existencia” (Rojas, 2006: 57). La presencia del austriaco se muestra desde el primer número de la revista La Torre con el artículo "La forma interior de la literatura europea" (1953). Después de citar una de las obras fundamentales sobre el análisis de la literatura europea y su relación con la literatura romana -nos referimos a la importante obra de Ernst Robert Curtius, Europäische Literatur and Lateinisches Mittelalter (ibid.: 99)-, Schajowicz reflexiona sobre la relación entre la tragedia griega y la tragedia 'moderna', mostrando el vínculo que permite seguir dotando de actualidad al gran género literario de la Grecia clásica. Para ello el siguiente texto es un buen exponente, en el que se sintetiza y expresa el humanismo de carácter clásico heredado al tiempo que se impregna de contemporaneidad y actualidad. La Antigüedad no es ya sólo un redescubrimiento, sino como dice el autor, es "una imagen latente" que el hombre occidental redescubre en sí mismo:

¿Pero es que existen, acaso, impulsos de la creación específicos del Occidente -aunque sean 'heredados' de la Antigüedad Clásica que tuvieran que objetivarse en obras poéticas totalmente diferentes de las otras culturas? A esto contestaría en sentido afirmativo. Uno de los mejores ejemplos podría suministrárnoslo el género de la tragedia, que, después de su nacimiento, plenitud y muerte en Grecia, volvió a surgir en el Occidente renacentista; éste lo 'aprendió en la escuela de Séneca, lo transformó (Marlowe, Shakespeare) cristianizándolo a veces (Calderón, Racine), y lo incorporó así a su acervo de tradiciones literarias. Pero la posibilidad de la existencia de la tragedia 'moderna' no se debe simplemente al redescubrimiento de este género dramático sino a una determinada predisposición en el hombre occidental, a la imagen latente que había en él de la realidad trágica, de repente desvelada por el nuevo contacto con la Antigüedad. (Schajowicz, 1953: 101-102).

\footnotetext{
${ }^{21}$ O más precisamente su propia cita: “Véase mi ensayo Orfeo y Anfión (La imagen del proto-artista en la poesía moderna), incluido en el $n^{\circ} 5$ de La Torre (Universidad de Puerto Rico, marzo de 1954), donde me refiero a los aspectos trágicos de la existencia del artista (pp. 51-52): 'Lo que éste siente como la más suyo no está sometido a su control, es un pasajero don de poderes celestes, quizás caprichosos y hasta maléficos desde la perspectiva del hombre. El artista es un condenado a la creación, ella le ofrece momentos de súbita euforia pero también le hace pasar por agonías lentas. Como un espectro, amenaza al creador la desesperanza por la lejanía de los dioses. Y hasta la presencia de ellos puede ser una prueba por encima de las fuerzas humanas. ¿No había advertido ya Apolo esta vulnerabilidad y esclavitud del hombre creador cuando se dirigió a las musas después de revelarse a Anfión? ¡Amadle, cuidadle! Pero sabed que para él / ya no hay felicidad... Sólo vive para mí. Le he escogido, como una cima es escogida por el rayo'" (Schajowicz, 1990: 182).
} 
Junto a la revista La Torre se inaugura el otro proyecto de la Universidad de Puerto Rico, la Biblioteca de Cultura Básica, en 1953 con Fausto de Goethe ${ }^{22}$. De la importancia de esta selección, Gregorio Weimberg ${ }^{23}$ escribirá una importante reseña sobre la colección cuatro años después en La revista, de la Biblioteca Nacional de Buenos Aires. Bajo el título “Biblioteca de Cultura Básica” (Weimberg, 1957) el profesor de la Universidad argentina resume lo poco común de las referencias de este carácter global que versan sobre colecciones de obras clásicas, circunscritas normalmente a determinados libros o autores. La razón de ocuparse de la colección en su conjunto se debe a las cualidades que impregnan la selección de cada uno de los textos que la componen y que explicita del siguiente modo: "En una palabra, los pobres clásicos son, quizá, lo peor tratados de toda la galería de autores"(Weimberg, 1957: 119), y esto a propósito de cómo, hasta la fecha, se habían ido traduciendo obras clásicas de unos idiomas a otros sin tener en cuenta el original o con estudios no coincidentes, textos incompletos y una suerte diversa de inexactitudes que descuidaban el rigor científico. En contraposición, la colección que dirige Ayala evidencia "una preocupación muy seria y un respeto encomiable por los quehaceres de la inteligencia", además de destacar "el valor de los títulos aparecidos hasta la fecha" (ibid.: 120). Posteriormente Weimberg centra su comentario sobre el Discurso del método, el Fausto, Los Lusíadas, El príncipe de Maquiavelo, las Lecciones sobre la filosofía de la historia universal de Hegel y las Obras completas de Shakespeare, destacando en cada una de estas obras tanto el valor de las traducciones elegidas, las más conocidas o eruditas hasta la fecha, como el de la calidad de los prólogos incorporados en cada una de estas ediciones. En definitiva, los libros que integran la colección de la Biblioteca de Cultura Básica son un hito en ese momento por su rigor científico. Destaca, por ejemplo, la traducción de la obra completa de Edgar Allan Poe encargada por Francisco Ayala a Julio Cortázar. En lo que refiere a obras fundamentales de Grecia y Roma, se publica La Ilíada de Homero y Las leyes de Cicerón. Ninguna de las dos obras está reseñada por Weimberg, pero curiosamente sí se las cita en la entrevista que el puertorriqueño Armindo Núñez realizó a Francisco Ayala sobre su estancia en Puerto Rico en 2008:

Uno de los elementos más significativos de su obra en Puerto Rico no meramente es la fundación y la marca de identidad de La Editorial, sino la importancia de los textos traducidos y la selección de traductores conocedores de su oficio tales como: Ildefonso Gil López (Las Lusíadas), Risieri Frondizi (El discurso del método), Luis Arocena (El príncipe), Luis Astrana (obras de Shakespeare), Roger Labrousse (Las Leyes), Luis Segalá Estalella (La Ilíada) y Antonio Espina (obras de Voltaire). (Núñez, 2008: 179) ${ }^{24}$.

\footnotetext{
${ }^{22}$ Precisamente son unos versos de Fausto de Goethe los que inician cada uno de los números de La Torre, con la siguiente traducción de Juan Ramón Jiménez: "Nací para ver, / mi sino es mirar; / jurado a mi torre, / el mundo me gusta. / Lo lejano miro, / miro lo cercano, / la luna y la estrella, / la selva y el corzo."

${ }^{23}$ Gregorio Weimberg (1919-2006) humanista y profesor de la Universidad de Buenos Aires, fue también director de la Biblioteca Nacional y director del Consejo Nacional de Investigaciones Científicas y Técnicas. Durante la dictadura sufrió asimismo la marginación, pero posteriormente reincorporado a la Universidad y reconocido con distintos honores, premios y distinciones.

${ }^{24}$ Y añade: "Esa primera colección de la que hablábamos, la Biblioteca de Cultura Básica, que todavía se sigue utilizando en muchos cursos universitarios ¿usted la concibió a raíz de unas conversaciones con don Jaime o usted traía ya la idea? No, todo fue surgiendo sobre la marcha creándose de un modo activo, espontáneo, personal, pero no como si fuera algo que lleva uno y lo saca en el momento para aplicarlo, sino que se iba
} 
A la intervención del periodista el intelectual granadino responde con un escueto "Sí". A continuación exponemos un análisis de la selección de la traducción y el estudio preliminar de que consta cada una de las dos obras incluidas en el catálogo de la colección. Actualmente ambas obras forman parte de los fondos de la Fundación Francisco Ayala en Granada (España), en un conjunto de diez que ha podido recuperar la institución ${ }^{25}$.

\section{La Ilíada de Homero por Guillermo Thiele y Luis Segalá}

La Ilíada de la Biblioteca de Cultura Básica consta de la conocida y prestigiosa traducción de Segalá, de 1908, que constituyó, junto con el resto de la obra de Homero, una de las cuatro partes de uno de los grandes hitos del homerismo en lengua española y que forma parte de las Obras completas de Homero publicadas en $1927^{26}$. El que fuera Catedrático de Griego de la Universidad de Barcelona desde 1906, Luis Segalá y Estalella (1873-1938) y que moriría en la Guerra Civil española en uno de los frecuentes bombardeos que sufrió la ciudad catalana, realizó una traducción de la obra de Homero caracterizada por una fidelidad absoluta al texto, de tal modo que hasta los años 80 se dice que Homero 'habló por boca de Segalá'. Carles Miralles en la semblanza que dedica al profesor de la Universidad de Barcelona señala la paradoja final de un Segalá olvidado después de su muerte, al tiempo que sus traducciones homéricas no cesaban de ser reeditadas incesantemente (Miralles, 2002: 13).

Antes de la fecha de 1956, año en que se publica esta Ilíada de la Biblioteca de Cultura Básica de la Universidad de Puerto Rico, al otro lado del Atlántico se publican las siguientes ediciones de las obras de Homero: la Odisea, también versión directa y literal del griego de Luis Segalá (Buenos Aires, Espasa-Calpe, 1951), y la conocida traducción de Alfonso Reyes de los IX primeros cantos de la Ilíada (México-Buenos Aires, Fondo de Cultura Económica, 1951). En la reseña de Jorge Páramo Pomareda (1954: 404) constatamos las siguientes publicaciones hispanoamericanas de tema

formando con la práctica. En esos años se crea la Facultad de Estudios Generales donde se ofrecen esos cursos de cultura básica para los estudiantes de primer año y simultáneamente usted labora en los comienzos de la misma. Con esa colección se están creando justamente los textos para los cursos que allí se ofrecen. Era la idea de Jaime Benítez a la que yo me sumé y cooperé con la mejor disposición de ánimo, así que fue un resultado del fruto de una colaboración intelectual amistosa" (Núñez, 2008: 173).

${ }^{25}$ Citamos a continuación el conjunto de obras que hasta 1957 se publicaron con el fin de contrastar la importancia de las dos obras del legado grecorromano en este corpus. Seguimos el estudio de Manuel Gómez Ros (2011: 259-260): “Goethe, Johann Wolfgang von, Fausto. Traducción de José Roviralta Borrell. Estudio de William Sinz. [Sin fecha; fue el primer volumen de la colección]; Hegel, Georg Wilhelm Friedrich, Lecciones sobre la filosofía de la historia universal, 1953. Traducción de José Gaos. Estudio preliminar de Adolfo G. Carpio; Descartes, René, El discurso del método, 1954. Traducción, estudio preliminar y notas de Risieri Frondizi; Camoens, Luís, Los Lusiadas, 1955. Traducción, prólogo y notas de Idelfonso Manuel Gil; Maquiavelo, Nicolás, El príncipe, 1955. Traducción de Luis Navarro. Edición, notas y apéndices de Luis A. Arocena; Shakespeare, William. Obras completas I. Macbeth, Trabajos de amor perdidos, Mucho ruido para nada, 1955. Edición y traducción de Luis Astrana Marín; Vega, Lope de, La Dorotea, 1955. Edición, prólogo y notas de José Manuel Blecua; Cicerón, Las leyes, 1956. Traducción, introducción y notas de Roger Labrousse; Homero, La Ilíada, 1956. Traducción de Luis Segalá."

${ }^{26}$ Con el subtítulo Versión directa y literal del griego por Luis Segalá y Estalella, edición adornada con ilustraciones arqueológicas de carácter homérico, Barcelona, Montaner y Simón editores, 1927. 
homérico anteriores a la de Alfonso Reyes, donde indica la ya existente edición de Segalá, que circulaba como 'pirata':

la Ilíada de Guillermo Jünemann (Concepción, Chile, 1902) y del Padre A. Lapalma (Buenos aires, s.f.), los fragmentos de la Ilíada y la Odisea traducidos por Leopoldo Lugones, la edición 'pirata' de un mal arreglo de la versión de Segalá y Estalella, debido a la Universidad Nacional de México (Ilíada, 2 vols.; Odisea, 1 vol., 192127), dos reimpresiones de la misma traducciones, una con introducción de Pedro Henríquez Ureña (Buenos Aires, 1943) y otra con estudio preliminar de Juan David García Bacca (Buenos Aires, Clásicos Jackson, 1943) y las Obras de Homero, comprendidos los Himnos (Pasto, 1938-9), traducidas por el colombiano Leopoldo López Álvarez.

El texto homérico está acompañado de una "Introducción" e "Índices" de Guillermo Thiele, profesor de la Universidad de Buenos Aires. La entidad del texto que sirve de introducción a la traducción de Segalá, será publicado en Caracas (1969) con el título Homero y su Ilíada, distinguido por el añadido de un único punto que lleva por título "La funesta mênis" (Thiele, 1969: 45-64). No obstante, no hay ninguna referencia a la edición previa de Puerto Rico. Como contraportada, leemos en la edición de 1969 el siguiente texto que destaca la importancia de su estudio:

Con su hondura y perspicacia habituales, el profesor Guillermo Thiele ha escrito una obra de síntesis, a la vez erudita y amena, que puede ser considerada, junto a los memorables trabajos de Alfonso Reyes, como la contribución más efectiva y perdurable hecha hasta hoy en nuestro idioma al estudio de la epopeya griega y de su más eximio representante. Se trata -como él mismo lo explica- de 'una breve excursión a través de ese laberinto que suele llamarse la cuestión homérica'.

En cuanto a los índices incluidos por Thiele, "Índices de nombres y de materias" (1956: 447-594), destacamos el rigor de la revisión a partir de los términos utilizados por Segalá. Con absoluto respeto a la edición del filólogo catalán, Thiele escribe (1956: 449-450):

Este índice reúne todos los nombres propios que se encuentran en la presente versión de la Ilíada, y en la forma en que Segalá y Estalella les dio. Les agrega algunos términos que el traductor vertió al castellano, mientras que la bibliografía internacional reciente suele referirse a ellos con la respectiva voz griega (p. ej.: Keres, véase Parcas; Litai, véanse Súplicas). Allí donde la versión deja lugar a dudas, el índice trata de desvanecerlas. [...] El índice pretende ser exhaustivo. Es decir, enumera, por regla, todos los pasajes en que aparece un nombre [...]. El índice está elaborado sobre la base del original griego. Como es imposible señalar, verso por verso, lo que le corresponde en el texto de Segalá y Estalella, el lector deberá guiarse por la numeración global al margen ${ }^{28}$.

\footnotetext{
${ }^{27}$ Páramo remite a la edición de 1927 de Segalá en la nota 2 de la reseña. Dice Segalá (1927: LXIV-LXV): "Algo escamados al ver que no se trataba de una nueva traducción, procuramos leer las dos páginas de dicha obra que se reproducían en reducido tamaño, y cuál no sería nuestro asombro al descubrir que la traducción publicada era una segunda edición de la nuestra, sin estampar en ella el nombre del traductor."

${ }^{28}$ Comprobamos de este modo cómo cumple la edición de esta Ilíada con las virtudes realzadas por Weimberg en su reseña sobre el conjunto de la colección dirigida por Francisco Ayala: -[ ] esta ejemplar empresa que es la -Biblioteca de Cultura Básica- patrocinada por la Universidad de Puerto Rico, además de los valores intrínsecos de sus publicaciones deberá servir de ejemplo para que en el futuro se abandone la poltronería intelectual, y se ponga cuidado en la selección de los textos, los que deberán estar acompañados, siempre, de estudios preliminares modernos, notas oportunas, ilustraciones adecuadas, índices completos, etc.- (Weimberg, 1957: 125).
} 


\section{Las leyes de Cicerón por Roger Labrousse}

La publicación de Las leyes de Cicerón en esta colección no es una casualidad. El interés del intelectual granadino por la obra del romano procede de al menos una década anterior a esta publicación de 1956, coincidiendo ya con su época de exilio. Así en Historia de la libertad (1943), texto ampliado posteriormente en "Ensayo sobre la libertad" (1944), leemos:

De esta nuevas corrientes de pensamiento, desarrolladas sobre todo en el campo de la antigua
cultura griega, representa en Roma un brote magnífico y precoz Cicerón -pensador muerto 43
años antes del nacimiento de Cristo-, que, en su escrito De las Leyes, se opone ya con vigorosos
argumentos a la vieja doctrina aristotélica de la desigualdad natural entre los hombres,
defendiendo la tesis de la igualdad de todos los humanos, fundado en la comunidad de la
naturaleza racional, de donde emana el derecho. Un siglo más tarde, Séneca, formado en la
filosofía estoica, proclama la igual capacidad de todos los hombres para la virtud, equiparando
de esta manera a libres y esclavos. (Ayala, 2006: 37).

Después de trece años, este interés ya manifiesto por la obra de Cicerón se materializa con la edición de Roger Labrousse que habría de servir para los estudios de la Universidad puertorriquense. Sobre la relación de Ayala con Labrousse, en Realidad (I, 4, 1947, 131-132) se reseña su libro En torno a la Teodicea (Edit. Facultad de Filosofía y Letras, Tucumán, 1946). Labrousse (1909-1956) es un intelectual de origen francés autoexiliado en 1939 con motivo del estallido de la Segunda Guerra Mundial. De México marcha a Argentina, instalándose en la Universidad de Tucumán como profesor en 1943. Allí se rodeará de un nutrido grupo de intelectuales, argentinos y otros igualmente exiliados de Europa por los conflictos bélicos, tales como Manuel García Morente, Rodolfo Mondolfo, Benvenuto Terracini, Elsa Tabernig o Jackques Rush. Catedrático de latín y literatura francesa, en palabras de Judith Casali de Babot, Labrousse fue "un erudito y sutil latinista, un profundo conocedor de la cultura clásica. La permanencia de su obra nos permite contar hoy con la impecable introducción, traducción e interpretación de Las leyes de Cicerón" (Casali, 2006: 116) ${ }^{29}$. Víctima a su vez de las nuevas injusticias totalitarias que sufrirá Argentina, será expulsado de su cátedra en 1953. Su restitución en 1956 llegará tarde, pues es el año en que muere, coincidiendo con la publicación en la colección Biblioteca de Cultura Básica de la Universidad de Puerto Rico, obra que sigue utilizándose como bibliografía en los planes de estudio de esta universidad.

La edición de Las leyes de Cicerón, junto con la de Álvaro D’Ors (1953) es una apuesta por dar a conocer esta obra del orador romano hasta entonces poco conocida

\footnotetext{
${ }^{29} \mathrm{O}$ también: “Estas reflexiones tomadas del pensador ruso - compartidas por Labrousse, no quedaron en meros análisis especulativos, las hizo parte de su vida, las volcó como docente, como investigador, como amigo, a través de su compromiso con la Universidad Nacional de Tucumán. De aquí su asombro e incomprensión y sus reiteradas notas solicitando una explicación cuando es dejado cesante en 1953, por iniciativa de Carlos Aguilar, golpe fatal para un hombre de su dedicación. Aunque continuara con sus Cursos en su casa y en el Círculo de la Prensa, esta medida privó a nuestra Universidad de una personalidad excepcional. Su reincorporación - mejor dicho la Resolución por la que se disponía su reintegración en Sociología, Cátedra que ni siquiera era la suya debido a la única partida existente-, llegó demasiado tarde e, irónicamente, de la mano de un gobierno militar que derribaba a un régimen constitucional, el de Perón, pero desnaturalizado por el autoritarismo y la arbitrariedad" (Casali, 2006: 123).
} 
fuera del ámbito jurídico. Ambos traductores, Labrousse y D’Ors, puntualizarán tal circunstancia en sus respectivas introducciones, señalando D'Ors que "el De legibus es una piedra fundamental dentro de la arquitectura de nuestra historia de Occidente" (1953: 9). Por su parte, Labrousse escribe que "El tratado de Las leyes (De legibus), de Marco Tulio Cicerón, es una obra importante y, sin embargo, mal conocida dentro de la literatura clásica latina", subrayando además que "de todos los trabajos doctrinales de Cicerón quizá sea éste el que permita apreciar mejor la flexibilidad y a veces la gracia del talento de su autor" (Labrousse, 1956: IX).

De la extensa introducción de la edición de Labrousse, muy destacables son las pinceladas que nos traslada sobre la figura de Cicerón, mostrándolo cercano y familiar en una comparación con el hombre contemporáneo, como ocurre con el retrato que nos hace de "El hombre Cicerón" (ibid.: XXIX-XXXII), del que destacamos el siguiente pasaje:

La vida de este hombre no fue la de un gran estadista, y tendremos que examinar la incidencia de sus limitaciones sobre su obra de pensador político. [...] Por otro lado, conviene interpretar con cierta prudencia las expresiones de los discursos y de los libros de Marco Tulio. Había dos registros en la vida romana, el privado y el público, y entre ellos la distancia era normalmente mucho mayor que en nuestra civilización. [...] Pensaban desde el comienzo y conservaban el hábito de pensar que el hombre que habla de toga no debe tener la sencillez y la familiaridad del hombre que abre su alma a un amigo. Tal vez el cristianismo, seguramente el romanticismo y la democracia moderna nos han sugerido otros usos: nos desnudamos por sinceridad y llamamos declamatorio a todo lo que parece indicar solemnidad en el estilo y en los sentimientos. Los romanos, que nos hubieran juzgado indecentes, consideraban, al contrario, que ciertas convenciones verbales eran imprescindibles para mantener la dignidad de la vida colectiva y preservar la herencia moral de los antepasados (mos maiorum). (Labrousse, 1956: XXIX-XXX).

Asimismo en esta introducción encontramos textos que dialogan entre el pasado clásico y el presente del traductor, como hemos comprobado en otros estudios incluidos en este trabajo. En este sentido, es particularmente destacable cuando, a propósito del libro I de Las leyes, Labrousse establece un diálogo entre el pensamiento de Cicerón y su contemporaneidad, donde la incorporación de otros textos del autor romano, en este caso procedentes de De finibus, deja abierto en el tiempo el campo de acción reivindicado para la humanitas:

Hemos recibido al nacer "una chispa de razón", pues "vemos en los niños como destellos de virtud, en los que debe encenderse la razón del filósofo para que ésta, como si anduviera bajo la conducta de un dios, alcance el fin último de la naturaleza" ${ }^{\prime 30}$. Pero sin el auxilio de una buena educación dejamos que se apague casi del todo. Por eso la sociedad general necesita el refuerzo de las sociedades particulares. No obstante, esto no significa que no ejerza influencia alguna en la vida del ciudadano. Al contrario, de la pertenencia a una misma especie deriva la virtud de humanitas, es decir, aquella que consiste en respetar a todo hombre por el solo hecho de que es nuestro semejante. (ibid.: LIX).

\footnotetext{
${ }^{30}$ Referencia en nota 52 a Fin V, 43.
} 


\section{CONCLUSIÓN}

Hemos expuesto en este trabajo la relación de Francisco Ayala con un grupo importante de intelectuales que publicaron en las revistas que dirigió en su exilio, primero junto con Lorenzo Luzuriaga en Realidad y luego en La Torre con Jaime Benítez como director. Traductores, helenistas y latinistas, dejaron en estos lugares documentos fundamentales de análisis y estudio de la Antigüedad para la civilización contemporánea. Junto con ello, tanto la selección de las obras como algunas cuestiones de análisis expuestas, revelan el interés por el humanismo de origen clásico. Desde la Paideía de Jaeger traducida por Wenceslao Roces, a Las leyes de Cicerón traducida y prologada por Roger Labrousse, hemos recorrido un itinerario que va del bienio 19471948 con Realidad, a los años 1953-1956 con La Torre, en el que la figura de Francisco Ayala permite que estos textos se difundan a través de su publicación en dos de las revistas más importantes en lengua española de ese momento ${ }^{31}$.

Entre las reseñas y artículos de Realidad se intercalan dos documentos sobre el humanismo, el conocido texto de Martin Heidegger y el de Karl Kerényi, evidenciándose un claro interés por su publicación paralela y contrastada. El texto de Kerényi, como filólogo de lenguas clásicas, incide en aspectos del humanismo en la Antigüedad, así como en el papel que debe desempeñar el filólogo contemporáneo ante un humanismo que no debe reducirse al estudio histórico. Detrás de ello, está el ejercicio del compromiso activo del hombre y no sólo el estudio del ser, ni siquiera como pudo promoverse en las antiguas Grecia y Roma.

En este contexto no sólo encaja la publicación de una nueva Ilíada de Homero como libro fundacional de la literatura occidental, sino también Las leyes de Cicerón. Una de las claves está en la referencia a la humanitas ciceroniana. Baste retomar una de las citas de otro de los textos de Cicerón que utiliza Labrousse, en concreto procedente de Los deberes (Labrousse 1956: LX): “Debemos asignarnos una sola meta: identificar el interés de cada uno con el de todos los demás" (Off.3.26-28). Esta frase de Cicerón parece resonar en todos los escritos aquí expuestos, en la selección de los textos, así como en las vidas exiliadas que pusieron en marcha toda una maquinaria de conocimiento y de estudio. La Antigüedad ya no es sólo un conocimiento heredado a través de diversos estratos de la historia y sus necesarias adaptaciones. La Antigüedad es recuperada en vivo y directo diálogo con el presente, como esencia que descubre el ser occidental exiliado dentro de sí -tal y como nos lo ha expuesto Schajowicz-, y que se suma al compromiso intelectual que hace de todos los autores aquí reseñados auténticos humanistas para la

\footnotetext{
${ }^{31}$ Para destacar la importancia de ambas revistas, con respecto a Realidad nos dice L. García Montero (2007: XXIX): “Realidad, publicada entre enero de 1947 y diciembre de 1949, fue una de las revistas más importantes de la cultura hispánica en su época. Sus responsables pudieron sentirse orgullosos en más de una ocasión de haber protagonizado un esfuerzo de alta calidad, con colaboraciones oportunas y muy significativas. Convocaron a lo mejor de la cultura de su tiempo para intentar comprender, diagnosticar y pensar en un mundo en crisis"; o, con respecto a La Torre, $\mathrm{M}^{\mathrm{a}} \mathrm{J}$. Sánchez Montes (2006: 97): "Durante los años que estuvo al cuidado de Francisco Ayala La Torre fue la mejor revista de su género en lengua española."
} 
segunda mitad del siglo XX. El concepto romano humanitas está presente en quienes, como Francisco Ayala, forjaron un espacio ensayístico y divulgativo al servicio de los mejores intelectuales e investigadores del momento. Intelectuales víctimas de una época totalitaria y bélica, pero conscientes de su labor pedagógica y cultural aliviada de servidumbres en el continente americano.

\section{BIBLIOGRAFÍA}

Ayala F. (1988): Recuerdos y olvidos, Madrid, Alianza.

Ayala, F. ([1943] 2006): Historia de la libertad, edición facsímil, Granada, Ayuntamiento de Granada [Buenos Aires, Atlántida].

Ayala, F. ([1992] 2006): El tiempo y yo, o el mundo a la espalda, Madrid, Comunidad de Madrid, Consejería de Educación-Visor libros.

Ayala, F. (2007): Obras Completas III. Estudios literarios, edición de Carolyn Richmond, Barcelona, Círculo de Lectores-Galaxia Gutemberg.

Casali, J., Jorrat, J., Lupiáñez, G. (2006): “Roger Labrousse: sus aportes historiográficos en el campo de la filosofía política”, en AA. DD. (2006), Actas del Primer Congreso sobre la Historia de la Universidad de Tucumán, Tucumán, Secretaría General de la UNT: 115-125.

Chicharro, A. (2006): El pensamiento vivo de Francisco Ayala, col. Alminares, Granada, Dauro.

Díaz Díaz, G. y Santos-Escudero, C. (1982): Bibliografía filosófica hispánica (1901-1970), Madrid, CSIC.

Eliade, M. (1954): “Los mitos en el mundo moderno”, La Torre, 6, 69-86.

Emiliozzi, I. (ed.) (2012): Francisco Ayala en La Nación de Buenos Aires, Valencia, PreTextos.

García Jurado, F. (2008): "Prólogo", en I. López Calahorro (2008) Francisco Ayala y el mundo clásico, Granada, Universidad de Granada: 17-24.

García Montero, L. (2007): “La aventura de pensar el mundo", introducción al facsímil de la revista Realidad, I, col. Facsímiles de Revistas Literarias, Sevilla, Renacimiento, XXIX-LXXV.

García Montero, L. (2009): Francisco Ayala. El escritor en su siglo, col. Los libros de la Estrella, Granada, Diputación de Granada.

Gómez Ros, M. (2011): “Francisco Ayala, editor”, en L. García Montero y M. Rodríguez Gutiérrez (eds.) (2011), De este mundo y los otros. Estudios sobre Francisco Ayala, Madrid, Visor Libros: 249-261.

Heidegger, M. ([1948] 2007: “Carta sobre el humanismo”, Realidad, vol. III, 7 y 9, 10911115 [1-25] y 1467-1491 [343-367].

Jaeger, W. ([1957] 1962): Paideía, México, Fondo de Cultura Económica. 
Jiménez Delgado, J. (1958): “Supervivencia de Cicerón,”, Helmántica, vol. 9, 30, 351-362. Kerényi, K. ([1948] 2007): "El humanista en la encrucijada”, Realidad, vol. III, 8, 12531263 [147-157].

López Calahorro, I. (2008): Francisco Ayala y el mundo clásico, Granada, Editorial Universidad de Granada.

López Calahorro, I. (2010): “La transformación del mito de Medusa en Francisco Ayala. De Ovidio a Lucano", en J. Luque, Ma Dolores Rincón e I. Velázquez (eds.) (2010), Dulces Camenae. Poética y poesía latinas, Jaén-Granada, Sociedad de Estudios Latinos.

Luzuriaga, L. ([1947] 2007): “Werner Jaeger, Paideía. Los ideales de la cultura griega. Fondo de Cultura Económica, México, 3 vol., 1942-1945”, Realidad, vol. I, 2, 347-349 [307-309].

Menéndez Pelayo, M. (1953): “Apuntes sobre el ciceronianismo en España y sobre la influencia de Cicerón en la prosa latina de los humanistas españoles", en Biblioteca Hispano-Latina Clásica: III, 177-271.

Miralles, C. (2002): Lluis Segalá. Semblança biográfica, Barcelona, Institut d'Estudis Catalans.

Mommsen, T. ([1945] 2006): El mundo de los Césares, traducción y prólogo de Wenceslao Roces, col. Conmemorativa 70 Aniversario, México, Fondo de Cultura Económica.

Mondolfo, R. ([1947] 2007): “Benjamín Farrington, Ciencia griega de Tales a Aristóteles. Colección Pingüino, edit. Lautaro, Buenos Aires, 1947", Realidad, vol. II, 6, 10291031 [431-433].

Mondolfo, R. (1954): “Séneca y la infinitud del progreso espiritual”, La Torre, 5, 63-74.

Mora, A. (2000): “La paideía republicana de Joaquín Xirau”, prólogo en R. Xirau (2000) Obras completas de Joaquín Xirau. Tomo III, vol. 2: Escritos sobre historia de la Filosofía, Madrid, Fundación Caja Madrid, Anthropos editorial.

Navajas, G. (2006): “La escritura de la libertad y la cultura industrial en Francisco Ayala", Hispania, vol. 89, 4, 702-717.

Núñez, A. (2008): “Esto de recordar a los 101 años”, La Torre, 48, 169-181.

Oviedo, G. (2006): "Rodolfo Mondolfo. Humanista de izquierda", Cuyo. Anuario de Filosofía argentina y americana, 23, 155-192.

Páramo, J. (1954): “La Ilíada de Homero. Traslado de Alfonso Reyes. Primera parte: Aquiles agraviado. México, Fondo de Cultura Económica, 1951. 243 págs.”, Thesaurus (Boletín del Instituto Caro y Cuervo), Tomo X, 404-408.

Pró, D. (1967): Rodolfo Mondolfo, 2 vol. Buenos Aires, Losada.

Rivaya, B. (2000): “Comunismo y compromiso intelectual: Wenceslao Roces" (con presentación a cargo de José María Laso), Papeles de la FIM, 14, 145-187, y 15, 160-165. 
Rojas Osorio, C. (2005): “La filosofía en Puerto Rico: los transterrados”, Revista Filosofía Universidad Costa Rica, XLIII, 108, 57-62.

Romero, F. (dir.) ([1947-1949] 2007): Realidad. Revista de ideas (Buenos Aires, 1947-1949), prólogo de L. García Montero, colección completa (18 números), col. Facsímiles de Revistas Literarias, Sevilla, Renacimiento.

Romero, J. L. ([1947] 2007): “Teodoro Mommsen, El mundo de los Césares. Ed. 'Fondo de Cultura Económica', México, 1945”, Realidad, vol. I, 1, 165-166 [147-148].

Romero, J. L. ([1948] 2007): “Ludwig Friedländer, La sociedad romana; Historia de las costumbres en Roma, desde Augusto hasta los Antoninos, Fondo de Cultura Económica, 1947", vol. III, 8, 1948, 1376-1378 [270-272].

Romero, M. (2005): “Traducciones y ediciones de la obra de Mommsen en España (1876-1905)", en J. Martínez-Pinna (coord.) (2005) En el centenario de Theodor Mommsen (1017-1903): homenaje desde la Universidad española, Málaga, UMA, Servicio de Publicaciones: 135-152.

Sánchez Guevara, O. (2011): “Ludwig Schajowicz, a un siglo de su nacimiento" (consultado en julio de 2012).

Sánchez Montes, M. J. (2006): “Ayala en Puerto Rico, Puerto Rico en Ayala”, en A. Sánchez Trigueros y M. A. Vázquez Medel (2006) Francisco Ayala y América, Sevilla, Alfar: 89-100.

Schajowicz, L. (1953): “La forma de la literatura europea”, La Torre, 1, 95-111.

Schajowicz, L. (1954): “Orfeo y Anfión”, La Torre, 5, 63-74.

Schajowicz, L. ([1962] 1990): Mito y existencia, Puerto Rico, Universidad de Puerto Rico.

Quiñónez, B. A. y Ale, M. C. (2009): “Los estudios clásicos en Tucumán”, Circe clás. mod. 13, 189-198 (consultado en julio de 2012).

Roces, W.: “Bibliografía traducida por Wenceslao Roces", Asociación Cultural Wenceslao Roces (consultado en julio de 2012).

Weimberg, G. (1957): “Biblioteca de Cultura básica”, La Biblioteca (Revista de la Biblioteca Nacional), Tomo IX, 1, Buenos Aires, 119-125.

Xirau, R. (1986): “Joaquín Xirau (1895-1946). Un esbozo” (consultado en julio de 2012).

\section{Ediciones de la Ilíada y la Odisea de Homero consultadas}

Homero (1951): La Ilíada. Primera parte: Aquiles agraviado, traslado de A. Reyes, México, Fondo de Cultura Económica.

Homero ([1951] 1998): Odisea, traducción de L. Segalá, introducción de A. López Eire, Madrid, Espasa Calpe.

Homero (1956): La Ilíada, traducción de L. Segalá, introducción e índices de Guillermo Thiele, Madrid, Ediciones de la Universidad de Puerto Rico, Revista de Occidente.

Homero (2010): Ilíada, prólogo, traducción y notas de O. Martínez García, Madrid, Alianza. 


\section{Ediciones de Las leyes de Marco Tulio Cicerón ${ }^{32}$ consultadas}

Cicerón, M. T. (1924): Obras completas de Cicerón. Tomo VI. Tratado de la República, Tratado de Las leyes, versión castellana de Francisco Navarro, Madrid, Biblioteca Clásica.

Cicerón, M. T. ([1953] 1970): Las leyes, traducción, introducción y notas por Álvaro D’Ors, Madrid, Instituto de Estudios Políticos.

Cicerón (1989): La República y Las leyes, edición de J. Mª Núñez González, Madrid, Akal. Cicerón (1956): Las leyes, edición bilingüe, introducción, traducción y notas de R. Labrousse, Madrid, Ediciones de la Universidad de Puerto Rico, Revista de Occidente.

Cicerón (1989): Las leyes, introducción, traducción y notas de R. Labrousse, Madrid, Alianza.

Cicerón, M. T. (2009): Las leyes, traducción, introducción y notas de Carmen Teresa Pabón, Madrid, Gredos.

${ }^{32}$ Sobre las iniciales del orador romano, “M. T" (Marco Tulio), las anotamos en los casos en que los autores de las ediciones las han hecho constar. 